\title{
Management of Children with Multiple Sclerosis
}

\author{
Anusha K Yeshokumar and Brenda Banwell \\ Children's Hospital of Philadelphia, Perelman School of Medicine, University of Pennsylvania, Philadelphia, Pennsylvania, US
}

he care of children and adolescents with multiple sclerosis (MS) requires appreciation of the impact of the disease on the developing brain and, in particular, the risk for cognitive impairment and academic challenges. Relapse rates in the first three years from onset are high, with an average of 1.5 relapses per year, and often require hospitalization for acute corticosteroid therapy. Disease modulatory therapies are typically prescribed, although formal clinical trials in the pediatric MS population are only just now being realized. In this review, we discuss strategies to optimize therapy for an individual child or teenager, including utilization of a multidisciplinary approach to care.

\section{Keywords}

Pediatric multiple sclerosis, immunomodulation, safety, efficacy, relapse, treatment

Disclosure: Anusha K Yeshokumar has no relevant conflicts of interest to declare. Brenda Banwell has received remuneration for work as a central MRI reviewer for Novartis. No funding was received in the publication of this article.

Compliance with Ethics: This study involves a review of the literature and did not involve any studies with human or animal subjects performed by any of the authors.

Authorship: All named authors meet the International Committee of Medical Journal Editors (ICMJE) criteria for authorship of this manuscript, take responsibility for the integrity of the work as a whole, and have given final approval to the version to be published. open Access: This article is published under the Creative Commons Attribution Noncommercial License, which permits any noncommercial use, distribution, adaptation, and reproduction provided the original author(s) and source are given appropriate credit.

Received: December 19, 2016

Accepted: February 7, 2017

Citation: US Neurology, 2017;13(1):42-6

Corresponding Author: Anusha K Yeshokumar, Division of Child Neurology, Children's Hospital of Philadelphia, Colket Translational Research Building, 10th Floor, 35th Street and Civic Center Boulevard, Philadelphia, PA 19104-4399, US. E: yeshokumaa@email.chop.edu
Multiple sclerosis (MS) onset during childhood or adolescence follows a relapsing-remitting pattern, with a high early relapse frequency, and is associated with a risk for cognitive impairment and future physical disability. Acute management of relapses, modulation of chronic disease course, cognitive evaluations and strategies for academic modifications, and multidisciplinary team approaches to the management of fatigue, bladder issues, and coping and emotional health are also key components of care. While the vast majority of MS patients experience onset in adulthood, up to $10 \%$ of patients experience symptom onset prior to 18 years of age. ${ }^{1,2}$

Relapses in pediatric MS patients, including the sentinel first "attack," are characterized by optic neuritis, transverse myelitis, brainstem syndromes, or rarely, an acute disseminated encephalomyelitis(ADEM) like (encephalopathy and polyfocal deficits) syndrome. By definition, an individual attack is defined by a new neurological deficit persisting more than 24 hours, and not better explained by another etiology, and occurring more than 30 days from the last recorded attack. ${ }^{3}$ MS diagnosis is confirmed by recurrent attacks and supported by magnetic resonance imaging (MRI) evidence for new lesions involving different regions of the central nervous system (CNS). The 2010 McDonald MS diagnostic criteria also allow for diagnosis to be made at the time of the first acute demyelinating attack if MRI demonstrates clinically silent lesions in two of the four regions typical for MS, at least one of which enhances with gadolinium. ${ }^{4}$ Application of the 2010 McDonald MS diagnostic criteria at the time of a first attack in a pediatric cohort requires consideration of the patient's age. When applied to patients over the age of 11 , the criteria very accurately identify children who later experience further MS attacks. Children who do not demonstrate the MRI features described in the 2010 McDonald criteria did not experience further disease and were not diagnosed with MS. ${ }^{5}$ In younger children, however, lesions are typically larger with less well-defined margins and may completely resolve. 6.7 The positive predictive value of the 2010 McDonald criteria at the time of a first attack is only $55 \%$, and thus caution must be used in applying the criteria in this age group, often requiring evidence of new disease over time on serial clinical and MRI examinations before confirming MS. ${ }^{8}$ Of note, a normal brain MRI at the time of a first demyelinating attack (in presentations of optic neuritis or transverse myelitis) strongly suggests a monophasic illness, with less than $3 \%$ of such children being diagnosed with MS over time. ${ }^{9}$

Over $90 \%$ of patients will experience near complete neurological recovery from relapses early in the disease. ${ }^{10}$ However, secondary disease progression, in which accrual of neurological disability occurs independent of relapses, may occur over time. While pediatric MS patients may take longer to develop secondary disease progression, they do so at a younger age than patients with onset in adulthood. ${ }^{11}$

Fundamental to the diagnosis of MS is the exclusion of other diagnoses. Progressive symptoms from onset would not be typical of the relapsing-remitting course seen in over $97 \%$ of pediatric MS patients and should lead to consideration of leukodystrophies, mitochondrial disease, compressive 


\begin{tabular}{|c|c|c|c|}
\hline Medication & Dosing Regimen & Potential Adverse Effects & Recommended Monitoring \\
\hline Glatiramer Acetate & $\begin{array}{l}20 \text { mg daily or } 40 \text { mg three times per } \\
\text { week (subcutaneous injection) }\end{array}$ & $\begin{array}{l}\text { Post-injection systemic flushing reaction, injection } \\
\text { site reactions (lipoatrophy) }\end{array}$ & None \\
\hline Interferon Beta 1a & $\begin{array}{l}30 \text { ug weekly (intramuscular injection) } \\
\text { or } 44 \text { mg three times per week } \\
\text { (subcutaneous injection) }\end{array}$ & $\begin{array}{l}\text { Flu-like symptoms, transaminase elevation, bone } \\
\text { marrow suppression, injection site reactions }\end{array}$ & $\begin{array}{l}\text { Monthly CBC and LFTs for the first } 6 \text { months, then } \\
\text { every } 6 \text { months; yearly thyroid studies }\end{array}$ \\
\hline Interferon Beta 1b & $\begin{array}{l}250 \text { ug every other day (subcutaneous } \\
\text { injection) }\end{array}$ & $\begin{array}{l}\text { Flu-like symptoms, transaminase elevation, bone } \\
\text { marrow suppression, injection site reactions }\end{array}$ & $\begin{array}{l}\text { Monthly CBCs and LFTs for the first } 6 \text { months, then } \\
\text { every } 6 \text { months; yearly thyroid studies }\end{array}$ \\
\hline Natalizumab & $\begin{array}{l}\text { 3-5 mg/kg (maximum } 300 \text { mg) every } 4 \\
\text { weeks (intravenous infusion) }\end{array}$ & Hypersensitivity reaction, PML & $\begin{array}{l}\text { JC virus serologies prior to treatment initiation and } \\
\text { then every } 3 \text { months }\end{array}$ \\
\hline Cyclophosphamide & $\begin{array}{l}\text { Induction and maintenance protocols } \\
\text { (intravenous infusion) }\end{array}$ & $\begin{array}{l}\text { Vomiting, amenorrhea, transient alopecia, } \\
\text { osteoporosis, sterility, increased infections, and } \\
\text { secondary malignancy (bladder cancer) }\end{array}$ & $\begin{array}{l}\text { CBC prior to and } 10 \text { days after each infusion, } \\
\text { urinalysis prior to each infusion }\end{array}$ \\
\hline Fingolimod & 0.5 mg daily (oral) & $\begin{array}{l}\text { Risk of bradycardia with first dose; macular edema, } \\
\text { increased infection risk (case reports of PML) }\end{array}$ & $\begin{array}{l}\text { CBC, Iymphocyte panel, LFTs, varicella serologies } \\
\text { prior to first dose; repeat CBC, Iymphocyte } \\
\text { panel, and LFTs every } 6 \text { months; ophthalmologic } \\
\text { evaluation prior to first dose then every } 3-6 \text { months, }\end{array}$ \\
\hline Teriflunomide & 7 mg or 14 mg daily (oral) & $\begin{array}{l}\text { Transaminase elevation, alopecia, increased } \\
\text { infections; teratogenicity (pregnancy category X) }\end{array}$ & $\begin{array}{l}\text { Pregnancy test, CBC, LFTs, and tuberculin skin test } \\
\text { prior to tretament initiation; Monthly LFTs for the } \\
\text { first } 6 \text { months, then every } 6 \text { months }\end{array}$ \\
\hline Dimethyl Fumarate & $\begin{array}{l}\text { 120mg or 240mg divided into two doses } \\
\text { daily (oral) }\end{array}$ & $\begin{array}{l}\text { GI symptoms (nausea, vomiting, and/or abdominal } \\
\text { pain), Iymphopenia, case reports of PML }\end{array}$ & $\begin{array}{l}\text { CBC and lymphocyte profile prior to treatment } \\
\text { initiation, then } \mathrm{CBC} \text { every } 6 \text { months }\end{array}$ \\
\hline Rituximab & $\begin{array}{l}375 \text { mg/m2/dose weekly for four doses or } \\
750 \text { mg/m2/dose every } 2 \text { weeks for two } \\
\text { doses followed by maintenance protocol } \\
\text { (intravenous infusion) }\end{array}$ & $\begin{array}{l}\text { Hypersensitivity reaction, increased infections; } \\
\text { case reports of PML in rheumatologic conditions }\end{array}$ & $\begin{array}{l}\text { Hepatitis serologies, CBC, lymphocyte panels prior } \\
\text { to treatment initiation; interval of dosing dependent } \\
\text { on results of lymphocyte panels }\end{array}$ \\
\hline
\end{tabular}

CBC = complete blood counts; JC = John Cunningham VIRUSLFT = liver function tests; PML = progressive multifocal leukoencephalopathy

myelopathy, or rarely, severe nutritional deficiencies. ${ }^{12,13}$ Infectious etiologies must be excluded, particularly at the time of acute presentation and in all children with an ADEM-like first event.

\section{Immunomodulatory therapies for multiple sclerosis}

In the last several years, an increasing number of treatment options have been made available for the treatment of MS. There are currently ten medications that have been approved by the US Food and Drug Administration (FDA) for use in adults. There are currently no medications that have been approved by the European Medicines Agency (EMA) or the FDA for use in children, however, treatment studies in pediatric MS are currently underway as discussed below. Here, we review many of the medications that have been used off-label in pediatric MS (see Table 1). Information on their efficacy, tolerability, and safety in children has mostly resulted from anecdotal experience and retrospective analyses. The most commonly used treatments for MS in children include interferon beta and glatiramer acetate. These medications were initially approved for use in adults in the 1990s and have a favorable safety profile in children and adults. These medications are injectables (either subcutaneous or intramuscular), and many children struggle with adherence to frequent needles.

\section{Glatiramer acetate}

Glatiramer acetate is a heterogenous mixture of synthetic polypeptides found in myelin basic protein. It is thought to act as an immunomodulatory agent by inhibiting specific regulatory and effector T-cells and by altering function of antigen-presenting cells. ${ }^{14,15}$ Glatiramer acetate is administered as a subcutaneous injection dosed at $20 \mathrm{mg}$ daily or $40 \mathrm{mg}$ three times a week. There is no reduction in dose required for children. Small retrospective studies in children have shown that glatiramer acetate is overall very well tolerated with no concerning or lasting side effects, even when used long-term. ${ }^{16,17}$ Patients may experience a transienthypersensitivity reaction with flushing and tachycardia lasting 30-90 seconds. These reactions are not dangerous and typically only occur a few times. Injection site skin reactions, particularly persistent skin dimpling (lipoatrophy), can occur. Careful teaching about injection technique is essential.

\section{Interferon beta}

Interferon beta-1a (Avonex ${ }^{\circledR}$, Biogen, Massachusetts, US; Rebif ${ }^{\circledR}$, EMD Serono, Inc., Massachusetts, US) and interferon beta-1b (Betaferon ${ }^{\circledR /}$ Betaseron ${ }^{\circledR}$, Bayer, Leverkusen, Germany) have all been administered to children with MS. Interferon beta medications are thought to work by modifying specific receptors that regulate immune signalling cascades, thereby inhibiting autoreactive $T$ cells and pro-inflammatory cytokines as well as inducing anti-inflammatory mediators. ${ }^{18}$ Medication selection within this class can be guided by administration preferences and after consideration of side-effect profile. Avonex is administered as a $30 \mathrm{ug}$ intramuscular injection once a week, Rebif as a 22-44 ug subcutaneous injection three times a week, and Betaferon/Betaseron as a $250 \mathrm{ug}$ subcutaneous injection every other day. Medications are typically slowly up-titrated to full dose over four to six weeks to minimize side effects.

Studies in adults with MS have demonstrated that interferon beta medications can reduce the frequency of relapses and clinical/radiographic disease activity in up to one third of patients. These medications are generally 
very well tolerated, although side effects can include flu-like symptoms in the days after injections, injection site skin reactions, headaches, muscle aches, fatigue, leukopenia, anemia, thrombocytopenia, increased liver enzymes, and thyroid dysfunction. The flu-like symptoms and other symptoms occurring following injection can be prevented and treated with the use of over-the-counter non-steroidal anti-inflammatory medications. Blood monitoring should including performance of complete blood cell counts, liver function tests, and thyroid function tests monthly for the first six months and then every six months subsequently (as side-effects are less likely to occur the longer a patient has been on the medication). If liver function tests become elevated, a reduction in the dose with subsequent slow re-uptitration can be done, or another medication can be considered. ${ }^{19}$

\section{Higher-potency therapies}

A second-line treatment must often be considered in patients with MS because of intolerable first-line medication side effects or because of treatment failure, defined as continued clinical and radiographic relapses while on first-line medication. A study of 258 children with MS showed that nearly all were treated initially with interferon beta or glatiramer acetate, and that almost half changed medications during the course of the study (mean observation period of almost four years)..$^{20}$ Changes in medications require careful counseling and consideration of safety given risks of infection (including the often fatal disease, progressive multifocal leukoencephalopathy [PML]). For many of these therapies, safety in pregnancy remains unknown. Counseling regarding safe sexual practice with contraception and cessation of medication during pregnancy should be considered.

\section{Natalizumab}

Natalizumab is a monoclonal antibody that targets the a4b1-integrin and 4 b7 integrin molecules on vascular endothelium involved in migration of $\mathrm{T}$ and $\mathrm{B}$ lymphocytes across the blood-brain barrier. ${ }^{21}$ This medication therefore works in MS by preventing $T$ and $B$ lymphocytes from crossing into the CNS, resulting in one of the highest rates of relapse reduction in adults with $\mathrm{MS}^{22}$ It is administered as a once-per-month infusion of 3-5 $\mathrm{mg} / \mathrm{kg}$ (maximum dose of $300 \mathrm{mg}$ ). Retrospective studies in children have demonstrated an impressive suppression of clinical relapses, as compared to pre-treatment relapse frequency. ${ }^{23-26}$

Infusions of natalizumab are generally extremely well tolerated, and the most concerning side effect and biggest limitation of its use is concern for PML. PML is a potentially fatal brain infection caused by reactivation of the John Cunningham (JC) virus, a polyomavirus. Because of decreased lymphocyte migration into the brain in patients on natalizumab, unopposed $\mathrm{JC}$ virus infection can cause destruction of oligodendrocytes and multifocal demyelination. ${ }^{27}$ Risk factors for the development of PML include prior exposure to JC virus, prolonged duration of natalizumab treatment, and previous exposure to other immunosuppressive medications (for MS or comorbid autoimmune conditions). To assess for prior exposure to JC virus, serum titers are followed serially. While JC virus seropositivity is noted in 70\% of adults at the time of MS onset, ${ }^{28,29}$ lower age-dependent rates are seen in children. ${ }^{30}$ Because primary infection with JC virus occurs during childhood and adolescence, screening for $\mathrm{JC}$ virus exposure is recommended every three months in patients who are initially JC virus naïve. Higher JC virus titers are also associated with increased PML risk in those patients who are JC virus exposed, and thus, antibody status is monitored every three months while on natalizumab as well.

\section{Cyclophosphamide}

Cyclophosphamide is an alkylating agent that causes broad immunosuppression with cytotoxic effects. Retrospective studies show decreased relapse rate and improvement in disability in the majority of children with MS. ${ }^{31}$ Side effects can be prohibitive, however, and include vomiting, amenorrhea, transient alopecia, osteoporosis, sterility, infections, and secondary malignancy including bladder cancer. These potential side effects significantly limit the use of cyclophosphamide for pediatric MS.

\section{Oral agents}

There are now three oral agents that have been FDA-approved for use in adults: fingolimod, teriflunomide, and dimethyl fumarate. There are currently trials assessing the efficacy and long-term safety profiles of each of these medications ongoing in children with MS.

\section{Fingolimod}

Fingolimod (Gilenya ${ }^{\circledR}$, Novartis AG, Basel, Switzerland) is an oral medication that was FDA-approved for the treatment of MS in 2010 that targets the sphingosine-1-phosphate receptor. It is administered as a $0.5 \mathrm{mg}$ tablet taken once daily. One study of 17 children with MS taking fingolimod followed on average for 8.6 months showed significant improvement in relapses with no side effects. ${ }^{32}$ A prospective, double-blind, randomized, active-controlled study is now enrolling to evaluate the efficacy and safety of fingolimod versus interferon beta in children with MS (PARADIGMS). Side effects include potential for severe bradycardia with first dose (must be administered in a monitored clinical setting) in $0.6 \%$ of patients, macular edema in $0.5 \%$ of patients, and neoplasm in $2 \%$ of patients. ${ }^{33}$ Case reports describing the development of PML in adults with MS on fingolimod have been reported. ${ }^{34,35}$

\section{Teriflunomide}

Teriflunomide (Aubagio ${ }^{\circledR}$, Sanofi Genzyme, Massachusetts, US) is an oral medication, FDA-approved for the treatment of MS in 2012, that inhibits a mitochondrial enzyme required for DNA replication, preferentially diminishing the activation and proliferation of fast-replicating cells, including T- and B-cells. It is administered as a 7 or $14 \mathrm{mg}$ tablet taken once daily. Studies in adults with MS have demonstrated that teriflunomide decreases relapse rate and the accrual of neurologic disability compared with placebo but not statistically more than interferons. ${ }^{36} \mathrm{~A}$ randomized, double-blind, placebo-controlled trial is now enrolling to evaluate the safety and efficacy of teriflunomide in children with MS (TERIKIDS). Side effects include alopecia in about $10 \%$ of patients and elevated transaminases and/or hepatotoxicity in about $15 \%$ of patients. ${ }^{37}$ Teriflunomide also has a black box warning for use in pregnancy due to risks of teratogenicity. A medication washout with cholestyramine or activated charcoal can be done in the event of pregnancy.

\section{Dimethyl fumarate}

Dimethyl fumarate (Tecfidera) is an oral medication that was FDAapproved for the treatment of MS in 2013. The exact mechanism of action remains unclear, but the medication has been shown to reduce cytokine production and lymphocyte count. ${ }^{38}$ It is administered as a 120 $\mathrm{mg}$ or $240 \mathrm{mg}$ tablet divided into two doses daily. There are currently multiple studies undergoing development or enrolling to evaluate the safety and efficacy of dimethyl fumarate in children with MS, including: an open-label, randomized, active-controlled study of dimethyl 
fumarate compared to interferon beta in children with MS (CONNECT); a randomized, placebo-controlled trial evaluating the efficacy and safety of dimethyl fumarate (IMAGINE) in children with MS; and an open-label study evaluating the effect of dimethyl fumarate on brain MRI lesions in children with MS (FOCUS). Side effects include gastrointestinal (GI) symptoms such as nausea, vomiting, and/or abdominal pain in about $30 \%$ of patients ${ }^{39}$ and lymphopenia in about $20 \%$ of patients..$^{40}$ Case reports describing the development of PML in adults with MS on dimethyl fumarate have been reported. ${ }^{14,42}$ Given the suspected association between lymphopenia and the development of PML, altered dosing or discontinuation may be considered if lymphopenia is to develop.

\section{Rituximab}

RituximabismonoclonalantibodythattargetstheCD20receptoronactivated B lymphocytes. It is not an FDA-approved medication for the treatment of MS but has been used off-labe| ${ }^{43,44}$ because of the extensive data available on its use in other autoimmune conditions in children. ${ }^{45.46}$ It is administered as an infusion dosed as one course every six months. A course can be administered as $375 \mathrm{mg} / \mathrm{m}^{2} /$ dose weekly for four doses or $750 \mathrm{mg} / \mathrm{m}^{2} /$ dose every 2 weeks for two doses. A study of eight children and adolescents with neuromyelitis optica and three with MS showed that nine patients experienced a reduction in relapses on rituximab. ${ }^{47}$ Infusions of rituximab are generally well tolerated, though allergic reactions can occur. PML has been reported in adults with systemic lupus erythematous receiving rituximab, ${ }^{48,49}$ though, as noted above, the risk may in part result from the prior use of other immunosuppressive medications in these patients. There have been no reports of PML reported in adults with MS. A very similar medication, ocrelizumab, has undergone large clinical trials demonstrating an impressive reduction in clinical relapses, new/enlarging lesions on MRI, and progression of disability. ${ }^{50,51} \mathrm{~A}$ pediatric MS trial is now being considered.

\section{Clinical trials in pediatric multiple sclerosis}

The EMA and FDA now require evaluation of all new therapies in children and, as discussed above, safety and efficacy of multiple medications for the treatment of MS are currently being evaluated in several pediatric trials. Conducting these trials can be challenging for several reasons. Pediatric MS remains a rare entity, and even with multi-site, collaborative studies, it can be difficult to enroll a sufficient number of patients to adequately investigate a medication's safety and efficacy. Ethical issues, including consideration of the appropriate control group and importance of patient assent, must be carefully addressed as well. The International Pediatric Multiple Sclerosis Study Group (IPMSSG), a group of collaborating international physicians and researchers who study pediatric MS, along with regulatory and industry stakeholders has established consensus regarding conducting clinical trials for pediatric MS..$^{52}$ The subsequently published guidelines standardize proposed trial designs including target subjects for enrollment, pharmacokinetic and pharmacodynamics evaluations, and measures to assess responsiveness (clinical markers, test batteries, and neuroimaging).

\section{The need for treatment algorithms}

While clinical trial level evidence for efficacy of MS therapies in children and adolescents would be of enormous value, it is also important to consider that very few MS patients (pediatric or adult) remain on one medication throughout their entire disease course. Defining adequate treatment response in a given patient, and addressing the individualized aspects of adherence and medication tolerability, necessitates a more global treatment approach that includes serial therapeutic options. In an attempt to standardize this process, the IPMSSG created guidelines for the assessment of treatment efficacy. After completion of 6 months on full dose medication with complete compliance, treatment failure has been defined as new T2 or contrasting lesions on MRI or two or more confirmed clinical attacks within one year. ${ }^{53}$ The IPMSSG is now designing more complex treatment strategies to aid clinicians and families through the many years of treatment facing children with MS. Key to these considerations is the need to have an age-span approach with partnerships with adult providers in order to evaluate the long-term impact of MS.

\section{Relapse management}

Close clinical and radiographic monitoring can identify relapses in MS. For most presentations, intravenous (IV) corticosteroids may be given, with the goal of accelerating the speed of symptom recovery. ${ }^{54,55}$ The proposed mechanism of action includes modification of cytokine responses, reduction of T-cell activation, and reduction of blood-brain barrier permeability such that entry of immune cells into the CNS is limited. IV methylprednisolone is dosed as 20-30 mg/kg (maximum $1 \mathrm{~g}$ ) daily for $3-5$ days, pending response. As long-term outcome is not changed with corticosteroid administration, relapses consisting of mild symptoms or not impairing daily function may be managed with close clinical monitoring.

When response to corticosteroids is insufficient or when corticosteroid therapy is contraindicated, intravenous immunoglobulin (IVIg) or plasma exchange (PLEX) may be considered. IVIg is thought to provide benefit by affecting cytokine production and T-cell proliferation in addition to binding to potential autoantibodies against myelin. It is administered as a $2 \mathrm{~g} / \mathrm{kg}$ course divided over two to five days. The use of IVIg in MS relapses has been limited to case reports and retrospective studies. ${ }^{56}$ PLEX is thought to provide benefit by removing circulating autoantibodies and immune complexes from the blood. A course of five to seven exchanges over 10-14 days has been described for MS relapses, particularly in patients with severe clinical syndromes such as brainstem or spinal cord involvement. ${ }^{57}$ Physical, occupational, and speech therapy consultations should be considered during a relapse to determine whether a patient would benefit from outpatient or inpatient rehabilitation.

\section{Symptomatic management and multidisciplinary care}

Children with MS often experience chronic symptoms akin to what can be seen in adults with MS, including fatigue, cognitive dysfunction, mood disturbances, neuropathic pain, spasticity, and bowel and bladder dysfunction. Careful attention should be paid to these symptoms, as effects can be seen on both school performance and quality of life. Lifestyle modifications should be considered, as appropriate, though escalation to pharmacologic or procedural interventions may be necessary. After improving sleep hygiene and initiating an exercise regimen, modafinil or amantadine can be considered for fatigue associated with pediatric MS. Diazepam, baclofen, and tizanidine may provide relief for spasticity, and gabapentin can provide neuropathic pain relief. Finally, anti-muscarinic agents, intradetrusor botulinum toxin A injections, and nerve stimulation/neuromodulation may provide benefit for bladder dysfunction. A multidisciplinary approach with collaboration from neurology, neuropsychology, social work, and 
physical and occupational therapy, along with other subspecialists as needed including psychiatry, rehabilitation medication, and urology, is essential for the care of children with MS.

\section{Summary}

Treatment options and approaches for pediatric MS is an evolving field of study. No medications have thus far been EMA-approved or FDA-approved for use in children. However, many medications used to treat adults with
MS are used off-label in children, and retrospective reviews have shown injectables and infused medications to be efficacious and well tolerated. Clinical trials examining newer agents are actively enrolling, though are challenged by the rarity of pediatric MS. Carefully considered strategies are now needed that address adequate and inadequate treatment responses, consideration of escalation to more potent treatments, safety (particularly as this relates to infection risks), and the impact of early treatment on cognition, health-related quality of life, and future disability. $\square$
1. Banwell B, Krupp L, Kennedy J, et al., Clinical features and viral serologies in children with multiple sclerosis: a multinational observational study, Lancet Neurol, 2007:6:773-81.

2. Chitnis T, Glanz B, Jaffin S, et al., Demo- graphics of pediatric-onset multiple sclerosis in an MS center population from the Northeastern United Sates, Mult Scler, 2009:15:627-31.

3. MCDonald WI, Compston A, Edan G, et al., Recommended diagnostic criteria for multiple sclerosis: guidelines from the International Panel on the diagnosis of multiple sclerosis, Ann Neurol, 2001;50:121-7.

4. Polman $\mathrm{CH}$, Reingold $\mathrm{SC}$, Banwell B, et al., Diagnostic criteria for multiple sclerosis: 2010 revisions to the McDonald criteria, Ann Neurol, 2011:69:292-302.

5. Kornek B, Schmitl B, Vass K et al., Evaluation of the 2010 McDonald multiple sclerosis criteria in children with clinically isolated syndrome, Mult Scler, 2012;18:1768-74

6. Banwell B, Shroff M, Ness J, et al., MRI features of pediatric multiple sclerosis, Neurology, 2007;68:S46-S53.

7. Chabas D, Castillo-Trivino T, Mowry EM, et al., Vanishing MS T2-bright lesions before puberty: a distinct MRI phenotype? Neurology, 2008:71:1090-3.

8. Sadaka Y, Verhey LH, Shroff MM, et al., 2010 MCDonald criteria for diagnosing pediatric multiple sclerosis, Ann Neurol, 2012;72:211-23. 9. Verhey LH, Branson HM, Shroff MM, et al., MRI parameters for prediction of multiple sclerosis diagnosis in children with acute CNS demyelination: a prospective national cohort study, Lancet Neurol, 2011;10:1065-73.

10. O'Mahony J, Marrie RA, Laporte A, et al., Recovery From Centra Nervous System Acute Demyelination in Children, Pediatrics, 2015;136:e115-123.

11. Renoux $C$, Vukusic $S$, Mikaeloff $Y$, et al., Natural history of multiple sclerosis with childhood onset N Eng/ J Med, 2007;356:2603-13.

12. O'Mahony J, Shroff M, Banwell B, Mimics and rare presentations of pediatric demyelination, Neuroimaging Clin NAm, 2013;23:321-36.

13. Miller $\mathrm{DH}$, Weinshenker $\mathrm{BG}$, Filippi $\mathrm{M}$, et al., Differential diagnosis of suspected multiple sclerosis: a consensus approach, Mult Scler, 2008:14:1157-74.

14. Weber MS, Hohlfeld R, Zamvill SS, Mechanism of action of glatiramer acetate in treatment of multiple sclerosis, Neurotherapeutics, 2007;4:647-53.

15. Lalive $\mathrm{PH}$, Neuhaus $\mathrm{O}$, Benkhoucha $\mathrm{M}$, et al., Glatiramer acetate in the treatment of multiple sclerosis: emerging concepts regarding its mechanism of action, CNS Drugs, 2011;25:401-14.

16. Kornek B, Bernert G, Balassy C, et al Glatirmaer acetate treatment in patients with childhood and juvenille onset multiple sclerosis, Neuropediatrics, 2003:34:120-6.

17. Ghezzi A, Therapeutic strategies in childhood multiple sclerosis, Ther Adv Neurol Disord, 2010;3:217-28.

18. Dhib-Jalbut $S$, Marks $S$, Interferon-beta mechanisms of action in multiple sclerosis, Neurology, 2010;74:S17-S24.

19. Tenembaum SN, Segura MJ, Interferon beta- 1a treatment in chilhood and juvenile- onset multiple sclerosis, Neurology 2006:67:511-3

20. Yeh EA, Waubant E, Krupp LB, et al., Multiple sclerosis therapies in pediatric patients with refractory multiple sclerosis, Arch Neurol,
2011;68:437-44.

21. Rice GP, Hartung HP, Calabresi PA, Anti-alpha4 integrin therapy for multiple sclerosis: mechanisms and rationale, Neurology 2005;64:1336-42.

22. Polman $\mathrm{CH}, \mathrm{O}$ 'Connor $\mathrm{PW}$, Havrdova $\mathrm{E}$, et al., A randomized, placebo-controlled trial of natalizumab for relapsing multiple sclerosis, N Engl J Med, 2006;354:899-910.

23. Huppke $P$, Stark W, Zurcher $C$, et al., Natalizumab use in pediatric multiple sclerosis, Arch Neurol, 2008;65:1655-8.

24. Ghezzi A, Pozzilli C, Grimaldi LM, et al., Natalizumab in pediatric multiple sclerosis: results of a cohort of 55 cases, Mult Scler 2013;19:1106-12.

25. Yeh EA, Weinstock-Guttmann B, Natalizumab in pediatric multiple sclerosis patients, Ther Adv Neurol Disord, 2010:3:293-9.

26. Kornek B, Aboul-Enein F, Rostasy K, et al., Natalizumab therapy for highly active multiple sclerosis, JAMA Neurol, 2013;70:469-75.

27. Stuve O, Marra CM, Jerome KR, et al., Immune surveillance in multiple sclerosis patients treated with natalizumab, Ann Neurol, 2006;59:743-7.

28. Padgett BL, Walker DL, Prevalence of antibodies in human sera against JC virus, an isolate from a case of progressive multifocal leukoencephalopathy I Infect Dis, 1973:127:467-70.

29. Shackelton $L A$, Rambaut $A$, Pybus $O G$, et al., JC virus evolution an its association with human populations, J Virol, 2006;80:9928-33.

30. Hennes EM, Kornek B, Huppke P, et al., Age-Dependent Seroprevalence of JCV Antibody in Children, Neuropediatrics, 2016:47:112-4.

31. Makhani N, Gorman MP, Branson HM, et al., Cyclophosphamide therapy in pediatric multiple sclerosis, Neurology, 2009:72:2076-82.

32. Fragoso YD, Alves-Leon SV, Barreira AA, et al., Fingolimod Prescribed for the Treatment of Multiple Sclerosis in Patients Younger Than Age 18 Years, Pediatr Neurol, 2015;53:166-8.

33. Khatri BO, Fingolimod in the treatment of relapsing-remitting multiple sclerosis: long-term experience and an update on the clinical evidence, Ther Adv Neurol Disord, 2016;9:130-47.

34. US Food and Drug Administration. FDA Drug Safety Communication: FDA warns about cases of rare brain infection with MS drug Gilenya (fingolimod) in two patients with no prior exposure to immunosuppressant drugs. Available at: www.fda.gov/Drugs/ DrugSafety/ucm456919.htm (accessed February 6, 2017)

35. Gyang TV, Hamel J, Goodman AD, et al. Fingolimod-associated PML in a patient with prior immunosuppression, Neurology, 2016;86:1843-5.

36. Xu M, Lu X, Fang J, et al., The efficacy and safety of teriflunomide based therapy in patients with relapsing multiple sclerosis: A meta-analysis of randomized controlled trials, J Clin Neurosci. 2016:33:28-31

37. Miller $A E$, Teriflunomide: a once-daily oral medication for the treatment of relapsing forms of multiple sclerosis, Clin Ther 2015;37:2366-80

38. Gross CC, Schulte-Mecklenbeck A, Klinsing S, et al., Dimethy fumarate treatment alters circulating $\mathrm{T}$ helper cell subsets in multiple sclerosis, Neurol Neuroimmunol Neuroinflamm. 2015:3:e183.

39. Phillips J, Selmaj K, Gold R, et al., Clinical significance of gastrointestinal and flushing events in patients with multiple sclerosis treated with delayed-release dimethyl fumarate Int J MS Care, 2015;17:236-43.

40. Longbrake $E$, Naismith $R$, Parks $B$, et al. Dimethyl fumarateassociated lymphopenia: risk factors and clinical significance Mult Scler J Exp Trans/ Clin, 2015.[Epub ahead of print].

41. Nieuwkamp DJ, Murk JL, van Oosten BW, et al., PML in a patient without severe lymphocytopenia receiving dimethyl fumarate, N Eng/ J Med, 2015;372:1474-6.

42. Rosenkranz T, Novas M, Terborg C, PML in a patient with lymphocytopenia treated with dimethyl fumarate, $N$ Engl J Med 2015;372:1476-8.

43. Milo R, Therapeutic strategies targeting B-cells in multiple sclerosis, Autoimmun Rev, 2016:15:714-8.

44. Salzer J, Svenningsson R, Alping P, et al. Rituximab in multiple sclerosis: A retrospective observational study on safety and efficacy, Neurology, 2016;87:2074-81.

45. Dale RC, Brilot F, Duffy LV, et al., Utility and safety of rituximab in pediatric autoimmune and inflammatory CNS disease, Neurology, 2014;83:142-50.

46. Nosadini M, Alper G, Riney CJ, et al., Rituximab monitoring and redosing in pediatric neuromyelitis optica spectrum disorder Neurol Neuroimmunol Neuroinflamm, 2016;3:e188.

47. Waubant $\mathrm{E}$, Graves J, Beres $\mathrm{SJ}$, Rituximab use in pediatric centra demyelinating disease, Pediatric Neurology, 2014:51:114-8.

48. Henegar CE, Eudy AM, Kharat V, et al., Progressive multifocal leukoencephalopathy in patients with systemic lupus erythematosus: a systematic literature review, Lupus, 2016:25:617-26.

49. Molloy ES, Calabrese CM, Calabrese $\mathrm{LH}$, The Risk of Progressive Multifocal Leukoencephalopathy in the Biologic Era: Prevention and Management, Rheum Dis Clin North Am, 2017:43:95-109.

50. Sorensen PS, Blinkenberg M, The potential role for ocrelizumab in the treatment of multiple sclerosis: current evidence and future prospects, Ther Adv Neurol Disord, 2016;9:44-52

51. Menge T, Dubey D, Warnke C, et al., Ocrelizumab for the treatment of relapsing-remitting multiple sclerosis, Expert Rev Neurother 2016;16:1131-9.

52. Chitnis T, Tardieu M, Amato MP, et al., International Pediatric MS Study Group Clinical Trials Summit: meeting report, Neurology, 2013;80:1161-8

53. Chitnis T, Tenembaum S, Banwell B, et al., Consensus statement: evaluation of new and existing therapeutics for pediatric multiple sclerosis, Mult Scler, 2012;18:116-27.

54. Barkhof F, Hommes OR, Scheltens P, et al., Quantitative MRI changes in gadolinium-DTPA enhancement after high-dose intravenous methylprednisolone in multiple sclerosis, Neurology, 1991:41:1219-22.

55. Filippini G, Brusaferri F, Sibley WA, et al., Corticosteroids or ACTH for acute exacerbations in multiple sclerosis, Cochrane Database Syst Rev, 2000;4:CD001331.

56. Spalice A, Properzi E, Lo Faro V, et al., Intravenous immunoglobulin and interferon: successful treatment of optic neuritis in pediatric multiple sclerosis, J Child Neurol, 2004:19:623-6.

57. Weinshenker BG, O'Brien PC, Petterson TM, et al., A randomized trial of plasma exchange in acute central nervous system inflammatory demyelinating disease, Ann Neurol, 1999;46:878-86. 